doi:10.12662/2359-618xregea.v9i2.p169-175.2020

\title{
ENSAIOS
}

\section{OS DESAFIOS DO PLANEJAMENTO DE PESSOAL EM NÍVEL GOVERNAMENTAL E O PORQUÊ DE O DFT NÃO SER A SOLUÇÃO MÁGICA}

\author{
THE CHALLENGES OF PERSONNEL PLANNING \\ AT GOVERNMENT LEVEL ANDWHY THE DFT \\ IS NOT THE MAGIC SOLUTION
}

\section{RESUMO}

Este ensaio tem como objetivo apresentar questões relevantes a respeito do Dimensionamento da Força de Trabalho (DFT) na Administração Pública Federal (APF). Para tanto, foi estruturado em três seções. A primeira expõe os conceitos sobre o tema e suas perspectivas de análise, destacando a relevância do Planejamento da Força de Trabalho (PFT) como estratégia ou diretriz governamental previamente à oferta e difusão dos modelos de DFT aos órgãos e às entidades da APF. Na segunda seção, apresenta-se uma síntese dos métodos de DFT sob ponto de vista estritamente organizacional, as recentes recomendações e diretrizes provenientes do Ministério da Economia (ME) e as indicações resultantes da convergência em literatura. Enfatiza-se que os modelos existentes possuem características prescritivas, oferecem variáveis relacionadas à mensuração de produtos ou entregas individuais e desconsideram características cognitivas do trabalho realizado em boa parte do setor público, tais como as atividades relativas à formulação e à implementação de políticas públicas. Por fim, propõe-se que os modelos prescritivos ou lineares sejam substituídos por modelos autóctones e previamente validados que considerem as características da organização - incluindo políticas, produtos ou serviços prestados à sociedade - e o nível de maturidade institucional para a adoção de novas técnicas e ferramentas de gestão. Afinal, são ferramentas e precisam conferir respostas mais rápidas aos anseios governamentais.

Palavras-chave: Dimensionamento da Força de Trabalho. Planejamento da Força de Trabalho. Administração Pública Federal.

Aleksandra Pereira Santos aleksandrapds@gmail.com Doutora em Psicologia Social. Pesquisadora da Universidade de Brasília. Brasília - DF - BR.

\section{ABSTRACT}

This essay aims to present relevant questions about Workforce Staffing in the Federal Public Administration. For this purpose, 
it was structured in three sections. The first section presents the concepts about the theme and its perspectives of analysis, highlighting the relevance of Workforce Planning as a government strategy or guideline prior to the provision and diffusion of theWorkforce Staffing models to APFagencies and entities. In the second section, a summary of the Workforce Staffing methods from a strictly organizational point of view, recent recommendations and guidelines from the Ministry of Economy and indications resulting from convergence in literature are presented. It is emphasized that thecurrent models have prescriptive characteristics, offer variables related to the measurement of products or individual deliveries, and disregard the cognitive characteristics of the work performed in a large part of the public sector, such as the activities related to the formulation and implementation of public policies. Finally, it is proposed that prescriptive or linear models be replaced by previously validated autochthonous models that consider the characteristics of the organization - includingpolicies, products or services provided to society - andthe level of institutional maturity for the adoption of new techniques and management tools. After all, they are tools and need to provide quicker responses to government concerns.

Keywords: Workforce staffing. Workforce Planning. Federal Public Administration.

\section{DIMENSIONAMENTO DA FORÇA DE TRABALHO: CONCEITOS E DILEMAS}

O Dimensionamento da Força de Trabalho (DFT) pode ser entendido como o processo que determina a quantidade de pessoas necessárias para uma determinada demanda ou restrição (LI; CHEN; CAI, 2007). Sob esse aspecto, trata-se de um termo cuja operacionalização é de natureza quantitativa, e seu foco está na estimativa de força de trabalho requerida em determinada unidade ou órgão. O DFT ganhou destaque no setor público nos últimos anos; em especial, por conferir racionalidade técnica para a identificação de necessidades de pessoal. Algo relevante no atual cenário fiscal e econômico. Nesse sentido, o presente ensaio tem como objetivo apresentar questões relevantes para o DFT na Administração Pública, tomando, como período de análise, dezembro de 2017, quando o Ministério da Economia (ME) estabeleceu critérios e procedimentos para a priorização do modelo referencial de gestão do DFT aos órgãos e às entidades do Poder Executivo.

No caso do setor público, em especial da APF, é possível falar em dois níveis de análise do DFT, uma vez que o processo ocorre em nível organizacional, que corresponde à necessidade de pessoal em ministérios, autarquias ou fundações, e em nível governamental, que se refere à estimativa de pessoal necessário para a APF, realizada pelo ME como órgão autorizador do provimento e ingresso de servidores públicos federais no âmbito do Poder Executivo.

Do ponto de vista organizacional, métodos e técnicas de DFT não são recentes. Entretanto, estavam concentrados em órgãos ou entidades das áreas de educação e saúde, conforme revisão de métodos de DFT realizado por Santos, Silva e Ferreira (2016). Tal percepção foi corroborada pelo $2^{\circ}$ Levantamento de Governança em Gestão de Pessoas ${ }^{1}$ realizado pelo Tribunal de Contas da União (TCU) em 2016, no qual foi identificado que somente $16 \%$ das organizações públicas pesquisadas utilizam, em estágio aprimorado, procedimentos técnicos para definir o quantitativo necessário de pessoal.

Adicionalmente, inexistia orientação quanto aos métodos de DFT que poderiam ser empregados pelos órgãos. Todavia, em dezembro de 2017, por meio da Portaria $n^{\circ} 477$, o ME estabeleceu critérios e procedimentos para a priorização do modelo referencial de gestão do DFT aos órgãos e às entidades integrantes do Sistema de Pessoal Civil da Administração Federal - SI-

1 Disponível em: https://portal.tcu.gov.br/unidades/ secretaria-de-fiscalizacao-de-pessoal/perfil-de-governanca-de-pessoas/ 
PEC. ${ }^{2}$ Para a implementação das diretrizes dispostas no normativo, foi celebrado termo de cooperação com a Universidade de Brasília (UnB) no valor de 6,5 milhões de reais. A Universidade será responsável pelo desenvolvimento de um modelo referencial, bem como pela transferência de tecnologia aos órgãos e às entidades do Governo. $\mathrm{O}$ normativo também vetou a realização de despesa para contratação, prorrogação ou substituição contratual relativas a DFT que não seja decorrente da aplicação da metodologia referencial. A metodologia encontra-se em fase de desenvolvimento e testes junto aos órgãos-piloto previamente selecionados pelo $\mathrm{ME}$.

O normativo, que parece suprir a carência metodológica previamente diagnosticada, ensejará desafios ao órgão autorizador de provimento de servidores públicos no âmbito da APF. À medida que os órgãos institucionalizam e expõem suas carências de pessoal baseados em uma metodologia do próprio $\mathrm{ME}$, haverá a necessidade de critérios objetivos a respeito de novas autorizações para concursos públicos que não sejam baseados, exclusivamente, na demanda oriunda dos órgãos ou nas pressões políticas decorrentes do ativismo de determinados dirigentes públicos. Isso porque, em cenários restritivos, será impossível aprovar todas as demandas de provimento resultantes da aplicação da metodologia. A autorização de novos concursos públicos já dá sinais de forte desaceleração confirmada pelas recentes declarações da equipe econômica do Governo.

Dessa forma, em nível governamental, não é possível separar a oferta de metodologia de DFT da necessidade de Planejamento da Força de Trabalho (PFT) no Governo Federal. Este último entendido pela International Personnel Management Association (IPMA) como o alinhamento estratégico do capital humano de uma organização com o direcionamento de suas operações. O processo metodológico de análise da força de trabalho em face dos desafios e da atuação do Estado, nos próximos anos,

2 Disponível em: https://conlegis.planejamento.gov.br/ conlegis/legislacao/atoNormativoDetalhesPub.htm?i$\mathrm{d}=14421$ \& tipoUrl=link pode ser resumido à seguinte questão: quantos e quais servidores, para qual Estado?

Sob esse aspecto, eleva-se a discussão de DFT ou PFT para um nível estratégico no qual questões como desestatização, terceirização e uso de tecnologias precisam ser colocadas em pauta. Dessa forma, faria sentido, mesmo após a aplicação de uma metodologia de DFT referenciada pelo $\mathrm{ME}$, autorizar o provimento de cargos para realização de atividades operacionais ou administrativas de baixa complexidade? Ou ainda, prover servidores com estabilidade em órgãos cuja ação do Estado se reduzirá ao longo dos anos e tenderá a ser assumida pela iniciativa privada?

Assim, considerando o contexto econômico do país, com a regra de teto de gastos e a quantidade de servidores públicos que se aposentará nos próximos anos - não apenas pelo alcance dos requisitos mínimos para aposentadoria, mas também pela reforma na previdência - o governo viverá um dilema (SCHETTINI; PIRES; SANTOS, 2018). Além de validar métodos e técnicas de DFT, precisará estabelecer um PFT para provimento de servidores públicos capaz de frear demandas. Tal planejamento precisa ser robusto e alinhado ao modelo de gestão fruto das transformações do perfil de atuação do Estado.

O DFT pressupõe o uso de técnicas quantitativas para estimar o número de servidores necessário, podendo ser utilizado em nível departamental, organizacional e - quiçá - governamental. O PFT, por sua vez, implica o uso de informações quantitativas, mas também qualitativas, relativas à força de trabalho, bem como prospecção de cenários futuros para o provimento e a locação de pessoal. Nesse aspecto, o ME editou a Portaria $n^{\circ} 193$, de 3 de julho de $2018^{3}$, que disciplina a movimentação para a composição da força de trabalho, previsto no $\S 7^{\circ}$ do art. 93, da Lei $n^{\circ} 8.112 / 1990$. A norma permite mobilidade e aproveitamento

3 Disponível em: http://www.imprensanacional.gov.br/ materia/-/asset_publisher/Kujrw0TZC2Mb/content/ id/28503558/do1-2018-07-04-portaria-n-193-de-3-dejulho-de-2018-28503542 
dos servidores da administração direta e indireta com o objetivo de promover o dimensionamento da força de trabalho no âmbito do Poder Executivo Federal. Segundo o dispositivo legal, a movimentação ocorrerá no interesse da Administração, considerado o interesse, o perfil profissional e a capacidade de execução das atividades do servidor.

A despeito de apresentar relativa fragilidade jurídica, uma vez que, do ponto de vista hierárquico, a regulamentação de dispositivo previsto em Lei deva ocorrer por meio de lei ou decreto; ou do eventual conflito com normas superiores (leis ou decreto) de cargos ou carreiras específicas. A Portaria $n^{\circ} 193 / 2018$ pode ser um excelente instrumento para o provimento de pessoal, sem ampliação de gastos, desde que seja utilizada a partir de um PFT racionalmente estruturado.

\section{DOS PRINCIPAIS MÉTODOS DE DFTE SUAS APLICAÇÕES NA GESTÃO ORGANIZACIONAL}

Apesar de discutidos há mais de 40 anos na literatura de gestão de pessoas (VIANNA et al., 2013), os métodos e as técnicas de DFT no setor público são limitados e escassos (ISIDRO-FILHO; SERRANO, 2016). Adicionalmente, Santos, Silva e Ferreira (2016), em revisão nacional e internacional sobre métodos e técnicas de DFT, identificaram métodos quantitativos avançados, todavia limitados às áreas de educação e saúde.

$\mathrm{Na}$ iniciativa privada, o DFT está alinhado à maximização de lucros e ao quantitativo de trabalhadores. Mesmo em literatura recente, ainda é definido com base em métodos que remontam a administração científica preconizada no modelo Taylorista. Tais metodologias, conforme os achados de Santos, Silva e Ferreira (2016), buscam realizar a avaliação dos tempos e movimentos gastos para o desempenho de determinada tarefa no intuito de identificar uma sequência mais apropriada e eficiente, bem como o quantitativo ideal de empregados.

Os métodos iniciais, no setor privado e público, evoluíram para os mais difundidos em termos organizacionais e, consequentemente, empregados no setor público. Entre esses, é possível destacar: o método de Gaidzinski (1998) aplicado de forma predominante na definição do quantitativo de enfermeiros necessários às instituições hospitalares e o método da Teoria das filas, modelo matemático simplificado que possui, entre as variáveis, o ritmo médio de chegada do usuário, a quantidade de profissionais, o ritmo médio de atendimento no sistema e o tempo médio de permanência na fila. Esses métodos preconizaram ulteriores, cuja característica central está na seleção de variáveis organizacionais por meio de um modelo matemático ou estatístico de regressão linear (MARINHO; VASCONCELOS, 2007; ISIDRO-FILHO; SERRANO, 2016).

Outra vertente metodológica, percebida em DFT, é a adoção da ferramenta gerencial de mapeamento de processos cujo objetivo é identificar os fluxos de atividades ou tarefas, as capacidades existentes, as competências e os recursos necessários aos principais processos organizacionais. Dessa forma, um subproduto dessa técnica é a identificação do quantitativo de empregados necessários em cada processo mapeado. Como limitação, convém considerar que o tempo dispensado no mapeamento de processos e seus custos, vis-à-vis as transformações ocorridas nos processos de trabalho, tornam-no, muitas vezes, obsoleto tão logo concluído.

Franco, Iwama e Serrano (2018) apresentam os três principais passos para a adoção de um método adequado de DFT:

a) a definição de uma teoria e análises organizacionais;

b) a escolha de variáveis relevantes e;

c) o uso da literatura científica. Pode-se afirmar que a centralidade na adoção ou definição de uma metodologia para DFT é a seleção das variáveis constituintes.

A despeito desse ponto, há que se considerar: a parcimônia, a qualidade e a confiabilidade das informações obtidas, os procedimentos utilizados em coleta de dados e a importância 
atribuída pela organização às variáveis selecionadas (FRANCO; IWAMA; SERRANO, 2018; SANTOS; SILVA; FERREIRA, 2016).

A metodologia referencial para a APF proposta por Serrano e Franco (2018) em aplicação/customização em organizações-piloto apresenta, como variáveis, entregas, esforços, quantidade de servidores lotados em determinada unidade/organização, carga horária de trabalho, quantidade de faltas, quantidade de horas de atividades educativas, quantidade de horas adicionais de trabalho e percentual de tempo produtivo. No que se refere às variáveis, Serrano et al. (2019) argumentam que, muitas vezes, as organizações não sabem quais variáveis são mais relevantes para mensuração, pois inexistem medidas sobre a produção e as informações sistematizadas. De toda forma, o modelo referencial parte da premissa de que o DFT deve considerar tanto a produção quanto a capacidade produtiva das unidades organizacionais e, nesse ponto, parece aproximar-se de metodologias típicas do setor privado nas quais a mensuração de resultados, de produção e entregas pode ser realizada de forma objetiva.

Considerando o quantitativo, a complexidade de atuação e os diferentes níveis de maturidade institucional dos órgãos e das entidades que compõem o Poder Executivo Federal, não é equívoco afirmar que haverá uma quantidade significativa de "esforço público" não mensurável por meio das variáveis propostas no modelo. De toda forma, o DFT pode subsidiar a tomada de decisão relativa à movimentação interna de força de trabalho ou solicitação de concursos públicos. Adicionalmente, Serrano et al. (2019) elencam que o DFT pode:

a) subsidiar a necessidade de formulação e entrega de programas e ações de capacitação e desenvolvimento, servindo seus resultados de contraste para as solicitações de eventos instrucionais por parte dos servidores e gestores;

b) complementar o processo de gestão do desempenho quando assentado principalmente na perspectiva com- portamental, podendo os resultados ser usados como contraste para mitigar vieses de resposta em avaliações ou compor escores ampliados e;

c) prover insumos para a elaboração de programas de saúde e segurança dos servidores, visto que uma das variáveis do modelo reúne dados sobre absenteísmo e similares.

\section{CONSIDERAÇÕES FINAIS: ENFRENTANDO OS DILEMAS}

Pode-se afirmar que o cenário fiscal continuará impondo esforço e atenção para agenda de gestão de pessoas tão olvidada nas últimas décadas. No que diz respeito ao DFT, em relatório da Organização de Cooperação e de Desenvolvimento Econômico (OCDE, 2010) sobre a gestão de recursos humanos no governo brasileiro, foi identificado que o Brasil possuía informações disponíveis para alinhar custos e quantitativo de pessoal; no entanto, a discussão sobre força de trabalho estava posicionada em uma agenda bem menos estratégica. As iniciativas e as regulamentações recentes do ME apontam que houve um avanço da agenda.

A disponibilização de metodologia padrão de DFT para a APF adotará órgãos e entidades de racionalidade técnica para a identificação de suas necessidades de pessoal. Todavia, considerando a amplitude e a complexidade de atuação dos órgãos e das entidades do Poder Executivo, o nível de maturidade/capacidade institucional e as funções de formulação, implementação e avaliação de políticas públicas, é possível perceber que o método de referência possuirá aderência em organizações cuja oferta de produtos ou serviços à sociedade pode ser mensurada e terá dificuldades de implementação em organizações cujos servidores desenvolvem atividades e possuem natureza cognitiva, relacionadas ao apoio à formulação de políticas públicas. Além disso, novos modelos para DFT podem ser desenvolvidos e validados estatisticamente, uma vez que esses modelos não são generalizáveis, e o construto é um 
campo da ciência de domínio aberto, em constante evolução. Em suma, o modelo referencial - oriundo de empresas privadas ou públicas caracterizadas pela prestação direta de serviços - requer cuidados para evitar reducionismos e distorções, característicos da adoção ou cópia de modelos gerencialistas.

Dessa forma - e considerado a diversidade de métodos existentes em literatura e validados organizacionalmente - o mais adequado seria deixar de impor metodologia específica e conferir autonomia/capacidade para que as organizações apliquem ou desenvolvam métodos, desde que observados padrões mínimos de modelos estatísticos para DFT. Para tanto, há que se proceder em alterações pontuais, na portaria vigente, capazes de flexibilizar a metodologia referencial e permitir a inovação no uso dos métodos de DFT.

Defende-se que a lógica própria da APF implica o desenvolvimento de técnicas autóctones e incrementais. As condições ideais para o DFT dispostas em literatura ou em metodologias mais complexas são ideais e, na maior parte das vezes, distantes da dinâmica própria dos órgãos públicos.

Adicionalmente, os esforços do órgão central em gestão de pessoas poderiam consertar-se de imediato em uma análise situacional da força de trabalho na $A P F$, o que implica realizar um PFT com vistas a obter diretrizes para provimento ou movimentação interna de servidores públicos em curto e médio prazo. Aqui seria ingênuo defender critérios exclusivamente técnicos, decorrentes da aplicação de um método quantitativo. É necessário admitir que não há separação absoluta entre política e administração, sendo possível conciliar a provisão adequada da força de trabalho - em termos de quantitativo e perfil ideal - com o modelo de atuação do Estado, legitimado pelo governo.

\section{REFERÊNCIAS}

FRANCO, V. R.; IWAMA, G. Y.; SERRANO, A. L. M. O que é Dimensionamento da Força de Trabalho? In: SERRANO, A. L. M. et al. (org.).
Dimensionamento na administração pública federal: uma ferramenta do planejamento da força de trabalho. Brasília: Enap, 2018.

GAIDZINSKI, R. R. Dimensionamento de pessoal de enfermagem em instituições hospitalares. 1998. 118 f. Tese (Livre-Docência) - Universidade de São Paulo, São Paulo, 1998.

ISIDRO-FILHO, A., SERRANO, A. L. M. Dimensionamento da força de trabalho no setor público: modelo quali-quantitativo aplicado. In: CONGRESSO CONSAD DE GESTÃO PÚBLICA, 9., 2016, Brasília. Anais [...]. Consad, 2016.

LI, Y.; CHEN, J.; CAI, X. An integrated staff-sizing approach considering feasibility of scheduling decision. AnnalsofOperationsResearch, v. 155, n. 1, p. 361-390, 2007.

MARINHO, B. L.; VASCONCELLOS, E. P. G. Dimensionamento de recursos humanos: desenvolvimento de um modelo conceitual e sua aplicação. REGE Revista de Gestão, v. 14, n. 2, p. 61-76, 2007.

ORGANISATION FOR ECONOMIC CO-OPERATION AND DEVELOPMENT (OCDE). Avaliação da Gestão de Recursos Humanos no Governo-Relatório da OCDE: Brasil: Governo Federal (Portugueseversion). OECD Publishing, 2010.

SANTOS, A. P.; SILVA, A. C. A. M.; FERREIRA, M. A. C. Um método simplificado para dimensionamento da força de trabalho na administração púbica federal. In: ENCONTRO NACIONAL DA ASSOCIAÇÃO NACIONAL DOS PROGRAMAS DE PÓS-GRADUAÇÃO E PESQUISA EM ADMINISTRAÇÃO, 40., 2016, Costa do Sauípe. Anais [...]. Costa do Sauípe: Enanpad, 2016.

SCHETTINI, B. P.; PIRES, G.; SANTOS, C. H. Previdência e reposição no serviço público civil federal do poder executivo: Micros- 
simulações. Texto para Discussão, nº 2365. Brasília: Instituto de Pesquisa Econômica Aplicada(IPEA), 2018.

SERRANO, A. L. M.; FRANCO, V. R. Metodologia e Método para Dimensionamento. In: SERRANO, A. L. M. et al. (org.). Dimensionamento na administração pública federal: uma ferramenta do planejamento da força de trabalho. Brasília: Enap, 2018.

SERRANO, A. L. M. et al. O Dimensionamento da Força de Trabalho como Ferramenta de Gestão. In: SERRANO, A. L. M.; MENDES, N. C. F.; ABILA, N. (org.). Dimensionamento na administração pública federal: uma ferramenta de gestão da força de trabalho. Brasília: Enap, 2019.

VIANNA, C. M. M. et al. Modelos econométricos de estimativa da força de trabalho: uma revisão integrativa da literatura. Physis: Revista de SaúdeColetiva, v. 23, p. 925-950, 2013. 